Aus der Königl. Universitäts-Frauenklinik zu München.

\title{
Beitrag zur Behandlung der Gesichtslagen durch manuelle Umwandlung in Hinterhauptslagen.
}

\author{
Von \\ Dr. B. Jungmann in Hamburg, \\ ehem. volontärarzt der Klinik.
}

Noch im Beginn unseres Jahrhunderts wurde die Gesichtslage von den Geburtshelfern als eine in jhren Folgen für Mutter und Kind höchst gefahrbringende und unter allen Umständen nur durch Kunsthülfe zu beendigende Lage angesehen. Man hat daher die verschiedensten Methoden ersonnen und empfohlen, die eine Verbesserung in der Haltung des Kindes herbeistrebten mittels Wendung, Zange oder auch durch manuelle Umwandlung in Schädellagen. Letztere Methode war insbesondere von Baudelocque warm empfohlen worden; wir werden auf sie noch weiter unten zu sprechen kommen. Jedoch wurden die Resultate eher schlechter als besser. Die mangelhaften Erfolge waren aber nicht nur der Methode selbst zuzuschreiben, sondern müssen hauptsächlich wohl aus zwei Umständen heraus erklärt werden: Erstens wurden ja derartige intrauterine Eingriffe nicht unter dem Schutze der Antiseptik ausgeführt, eine Thatsache, die um so sehwerer ins Gewicht fällt, je länger die Operation dauerte, die man ohne Narkose vornehmen musste. Zweitens kannte man den physiologischen Mechanismus der Geburt noch zu wenig, um zu wissen, dass nur die mentoposterioren Gesichtslagen eine absolut schlechte Prognose geben - die wenigen derartigen, durch die Naturkräfte oder Kunsthülfe glücklich beendigten Geburten, die in der Literatur bisher beschrieben sind, können hieran nichts ändern - während die andern häufig spontan verlaufen. Man ist in der Unkenntniss 
dieser Verhältuisse soweit gegangen, alle Gesichtslagen, also auch die mit dem Kinn nach vorn gerichteten, in Schädellagen zu verwandeln; doch war dies entschieden ein Fehler, demn es wurde aus der, wie wir heute wissen, prognostisch durchaus nicht ungünstigen mentoanterioren Gesichtslage dureh manuelle Umwandlung eine mindestens nicht günstigere Lage, nämlich die Vorderhauptslage.

Diese Polypragmasie wurde vollständig aufgegeben, nachdem Zeller und besonders Boer nachgewiesen hatten, dass Gesichtslagen nicht unbedingt zu den Dystokieen zu rechnen seien, sondern in der Mehrzahl der Fälle auch spontan mit glücklichem Ausgang für Mutter und Kind verlaufen können. So kam es denn, dass seit jener Zeit die manuelle Umwandlung von Gesichtslage in Schädellage nur einige wenige Male, so von Rosshirt in Tübingen 1842 und von Cazeaux in Paris 1858 ausgeführt wurde.

Erst die neuere grosse Statistik v. Winckel's (Pathologie der Geburt 1869) liess die Prognose der Gesichtslage doch ungünstiger erscheinen als bisher angenommen wurde; denn es „kamen bei den Geburten in Gesichtslage etwa 13 pCt., d. h. mehr als doppelt soviel todte und sterbende Kinder zur Welt, wie bei denjenigen in Schädellage." Die Gefahr liegt besonders darin, dass "durch die starke Anspanung des Halses ein Druck auf die Venae jugulares externae bewirkt wird; die hieraus resultirende Gehirnhyperämie kann sich zur Apoplexie steigern, wenn durch das Anstemmen des Kinnes unter dem Schossbogen auch die Venae jugulares internae comprimirt werden." Anch der Mutter drohen Gefahren; denm "durch die Verschiebung des Hinterhaupts wird die entsprechende Uterusseite stärker gespannt und dadurch die Sehmerzhaftigkeit der Wehen gesteigert; auch kann durch die scharfen Kanten des Gesichts eine beträchtliche Quetschung der mütterlichen Blase und Scheide bis zur Entstehung einer Blasenscheidenfistel bewirkt werden, ferner kann dadurch, dass die submentooccipitale Peripherie, welche durchtritt, nach den Messungen von Torggler immer grösser ist, als der kleine schräge, und zwar um 4,72 (der Durchmesser gegen den kleinen schrägen um 1,08-1,90), mithin der Damm bei Gesichtslagen mehr als bei Schädellagen gedehnt wird, leichter Dammrisse entstehen." So hat man denn erst in neuerer Zeit, angeregt durch die Empfehlung Schatz's (1873), sowie durch die Veröffentlichung Thorn's (1886), dem es gelang, in 24 Fällen $9 \mathrm{mal}$ aus einer Gesichtslage eine Hinterhauptslage mittels 
seiner neuen, combinirten Methode herzustellen, in einer Reihe von Fällen dieselbe versucht und mit Erfolg ausgeführt.

Noch in demselben Jahre publicirte Ziegenspeck einen Fall, in dem es ihm - unabhängig von Thorn - gelang; bei drohender Uterusruptur eine mentoposteriore Gesichtslage, nachdem die Baudelocque'sche und Schatz'sche Methode einzeln vergeblich versucht waren, durch Combination beider in eine 1. Sehädellage zu verwandeln, die er dann mit der Zange (Beckenausgang) glücklich beendete.

Aus der Berliner Poliklinik (Olshausen) berichtet Wullstein (1891) über 100 Fälle von Gesichtslage, in denen $10 \mathrm{mal}$ die combinirte Methode nach Schatz-Thorn versucht wurde. Nur 4 mal missglückte sie (einmal bei engem Becken), in den anderen 6 (auch hier einmal enges Becken) war der Erfolg ein guter: die Mütter blieben im Wochenbett fieberfrei und nur ein Kind wurde (aus unbekannter Ursache) todt geboren.

v. Weiss hat 20 Fälle von Gesichtslagen veröffentlicht, von denen Peters 13 an der Klinik von Braun nach der. Thorn'schen Methode $^{1}$ ) umzuwandeln versucht hat; und zwar gelang es ihm in 8 Fällen, Schädellage herzustellen, in der dann die Geburt meist rasch und spontan erfolgte; in einem Falle jedoch starb die Mutter am 9. Tage des Wochenbetts an eitriger Peritonitis, deren Ausgang von der Stelle, an welcher die Hand des Operateurs zwischen Uterusrand und Hinterhaupt vorgedrungen war, mit Sicherheit nachgewiesen werden konnte. Doch wäre es verkehrt, die Methode für den letalen Ausgang verantwortlich zu machen; ein Fehler in der Antisepsis hätte sich bei jeder gewöhnlichen innerlichen Untersuchung, die an der Kreissenden doch fast stets einen intrauterinen Eingriff darstellt, in gleich schwerer Weise rächen können!

Die übrigen 6 Fälle, die v. Weiss selbst operirt hat, können aber zu einer Beurtheilung der von Thorn empfohlenen Methode gar nicht herangezogen werden; denn in 6 Fällen hat er sich zwar auch eines combinirten, aber nicht des Thorn'schen, sondern des Baudelocque'schen Verfahrens bedient ( 2 mal mit, 4 mal ohne Erfolg). Und gerade der eine Fall, in dem er nach Thorn operirte (primäre Gesichtslage, deren Umwandlung in der Schwangerschaft nach der Schatz'schen Methode, damn während der Eröffnungs-

1) Nur mit der Abweichung, dass er sofort mit der inneren Hand das Hinterhaupt herunter holte. 
periode nach Thorn vergeblich versucht wurde), muss ebenfalls aus der Beurtheilung der Werthes der Thorn'schen Methodo ausscheiden, weil er, wie $v$. Weiss selbst zugiebt, wegen der bestehenden Cervixstrictur für die combinirte Umwandlung überhaupt ungeeignet war.

v. Steinbüche]. (Ueber Gesichts- und Stirnlagen, Wien 1894) stimmit mit Küstner ${ }^{1}$ ) darin überein, „dass, so ungefährlich die combinirten Handgriffe zur Umwandlung der Gesichts- in Hinterhauptslage von einem erfahrenen Geburtshelfer, unter strenger Beobachtung der modernen Antisepsis ausgeführt, auch sein mögen, dieser geringe Eingriff doch nicht gerechtfertigt werde durch das Minimum, um welches die Gesichtslage in Verlauf und Prognose ungünstiger ist als die Hinterhauptslage. Nur bezüglich der mentoposterioren Gesichtslage, also der complicirten Einstellung, kommt er ebenso wje v. Weiss zu dem Schluss, dass „ein Versuch diese ungünstige Einstellung durch combinirte Handgriffe (Thoru) umzuwandeln, nicht unberechtigt sei, insofern als dadurch dic erschöpfende, schwere Geburtsarbeit umgangen würde, welche bei mentoposteriorem Mechanismus entweder doch noch eine andere Kopfhaltung herstellt oder im ungünstigen Falle die Geburt in der erwähnten Einstellung vollendet."

Zur Beleuchtung dieser Ansichten möchte ich die beiden Fälle aus der jüngst von Gossmann veröffentlichten Arbeit citiren: „Im ersten (vor vielen Jahren behandelten) Falle handelte es sich bei normalem Becken um eine mentoposteriore Gesichtslage. Alle Versuche, durch Druck auf die Stirn das Kinn nach vorn zu bringen, scheitern. Daher exspectatives Verhalten. Nach einer Austreibungszeit von vollen 2 Tagen bei zeitweise erlahmender, doch immer wieder kräftig einsetzender Wehenthätigkeit tritt endlich das Kim tiefer und mehr nach vorn." Wegen Indication zur Beendigung der Geburt: Application der Zange: Das asphyktische Kind kann nicht mehr belebt werden; die Mutter trug ausgedehnte Druckbrandgeschwüre in der Scheide davon. Wochenbett fieberbaft. Gonesung. Im zweiten Falle (ebenfalls normales Becken) wandelte er die Gésichtslage nach Thorn (durch Umfassen des Hinterhauptes mit der Hand) in Hinterhauptslage um. Die Geburt beendigte er S. 722 .

1) Küstner, Müllers Handbuch der Geburtshilfe. 1889. Bd. II. 
nach 3 Stunden wegen Wehonschwäche mittels Zange mit glücklichem Ausgang für Mutter und Kind ${ }^{1}$ ).

Doch scheint die Reihe der bisher veröffentlichten Fälle noch zu klein zu sein, um die maassgebenden Schulen zu veranlassen, den seit $\mathrm{Bo}$ ër fast allgemein anerkannten möglichst exspectativen Standpunkt in der Therapie der Gesichtslagen zu verlassen und das neue Schatz-Thorn'sche combinirte Verfahren den geburtshülflichen Operationsmethoden einzureihen ${ }^{2}$ ).

Und so hoffe ich denn mit der Veröffentlichung dreier neuer Fälle von manueller Unwandlung von Gesichtslage in Hinterhauptlage, die ich an hiesiger Klinik und Poliklinik theils zu beobachten, theils selbst zu operiren Gelegenheit hatte, die Geburtshelfer auf diese aufmerksam zu machen und anzuregen, in einschlägigen Fällen die äusserst einfache Methode auf ihre praktische Verwendbarkeit selbst zu prüfen.

Bevor ich jedoch ihre Wirksamkeit an der Hand der drei Geburtsgeschichten beschreibe, will ich noch kurz die Entwicklungsphasen, aus denen sie sich herausgebildet, schildern, weil sich aus diesen die Vorzüge der neuen Methoden am besten ergeben.

Die älteste Methode, die von Baudelocque angegebene, ist eine combinirte und kann in zwei Modificationen ausgeführt werden:

1. Man geht mit der dem Kinn des Kindes entsprechenden, also bei erster Gesiehtslage mit der linken Hand in die Scheide ein, und sucht, während die äussere rechte Hand das Hinterhaupt kräftig tiefer drückt, mit zwei Fingern, die gegen das Kinu, den Oberkiefer, Stirn und Hinterhaupt angestemmt werden, das Gesicht in die Höhe zu schieben, der Brust möglichst zu nühern und das Hinterhaupt in das Becken zu leiten.

1) Hierzu muss ich bemerken, dass die Zange unter diesen Umständen - bei Wehenschwäche - nicht indicirt gewesen ist. Ja die Wehenschwächo bildet wegen der Gefahr der consecutiven Atonie des Uterus geradezu eine Contraindication zur Zange! Da der Kopf schon im Beckenausgang stand, so hiess es: entweder bei Wehenschwäche „Abwarten bis die Wehen besser werden"; oder bei Indication zur Beendigung der Geburt (z. B. Schlechtwerden der Herztöne): „Kristeller's oder Ritgen'scher Handgriff oder Combination dieser beiden Verfahren, wolche die Geburt sicher beendet und die Beckenausgangszange überflüssig gemacht hätten ".

2) Nur in der nenesten Auflage des vortrefflichen Doederlein'schen Werkes (Leitfaden für d. geburtshülfl. Operationscurs. 2. Auflage. Leipzig 1895) habe ich eine ausführliche und klarc Darstellung dieser Methode gefundell. 
Oder 2. man geht mit der dem Hinterhaupt der Frucht entsprechenden Hand in den Uterus ein, sucht dieses voll zu umfassen und ebenfalls wie in dom vorigen Verfahren durch eine Rotation des kindlichen Schädels um seinen Querdurchmesser in die FührungsJinie des Beckeneinganges einzustellen.

Doch ist dic Zahl der Fälle, die mittels dieser Methode erfolgreich behandelt wurden, eine sehr kleine. Der Hauptfehler, der jhr amhaftet, besteht darin, dass sie sich nur darauf beschränkt, ausschliesslich durch Manipulationen am kindlichen Schädel die abnorme Deflexionshaltung in die normale Beugehaltung umzuwandeln und dem innigen Zusammenlang zwischen der Anomalie der Gesichtseinstellung und der Haltung des kindlichen Rumpfes bezw. der Wirbelsäule gar keine Rechnung trägt.

Auf dieses Punctum saliens in der ganzen Lehre von der manuellen Correction der Gesichtslagen zuerst aufmerksam gemacht zu haben, ist das unbestreitbar grosse Verdienst Schatz's. Dieser hat durch die Mittheilung seiner ebenso sinnreichen wie einfachen Methode, die er mit gutem Erfolge allerdings nur in einem Falle angewandt hatte, diese Frage in neue Bahnen gelenkt.

Das von ihm empfohlene Verfahren geht von der mechanisch gut begründeten Ueberlegung aus, dass die Gesichtslage nur dann dauernd korrigirt bleiben köme, wenn auch die pathologische Sförmige Krümmung der Wirbelsäule in die normale Cförmige übergeführt sei. Doch ehe diese normale kyphotische Haltung. dor Frucht erreicht sei, müsse sie nothwendig eine Mittelstellung durchgemacht haben, die nur aus einer Verlängerung der langen Eiaxe resultiren könne. In der Beachtung dieses Momentes liegt der Werth der Schatz'schen Methode und zugleich der Schlüssel zur Erklärung einerseits der Wirksamkeit der später (1886) auf diesem Prinzip von Thorn construirten Methode und andererseits der Unwirksamkeit des Baudelocque'sehen Verfahrens. Denn bei dieser kommt es, da die Handgriffe nur am Kopfe wirken, njcht am Rumpf, nicht nur zu keiner Streckung der Eiaxe, sondern gerade zu einer Verkürzung, und gerade dieser Uebelstand, verstärkt durch den von der Uteruswand geleisteten Widerstand - den jedoch die Narkose beseitigen könnte - ist es, der die Correction der lordotischen Krümmung in die kyphotische verhindert.

Aus diesen Ueberlegungen heraus sehlug Sohatz vor, in der Wehenpanse die vordere Schulter des Kindes, also bei I. Gesichtslage die rechte mit der rechten Hand von aussen voll zu umfassen 
und unter gleichmässig starkem Druck nach dem Rücken des Kindes und etwas nach oben schieben, während zu gleicher Zeit von der anderen Hand der Steiss nach dem Fundus zu, dann nach der fötalen Bauchseite hin, schliesslich noch etwas nach abwärts gedrängt wird.

In der Ueberlegung, dass der alten Baudelocque'schen Methode gerade der Fehler anhafte, den die Schatz'sche corrigirt und dieser wieder der Vorzug jener abgeht, kam Thorn auf den glücklichen Gedanken, beide zu einer einzigen Methode zu combiniren, die er mit ausgezeichnetem Erfolge unter 24 Fällon 9 mal anwandte. Da dieselbe in ihren Componenten schon oben beschrieben ist, so brauche ich sie hier micht noch einmal zu schildern, zumal sie bei der nun folgenden Beschreibung unserer drei Fälle noch öfter angeführt wird.

I. Journ.-No. 699. Theres Zetterer, Dienstmagd, 22 J., IIp. Eintritt 21. IX. 95, 2 Uhr p. m.

Anamnese: Mutter der Kreissenden starb an unbekannter Krankheit, Vater und ein Bruder leben und sind gesund, 7 Geschwister starben in früher Jugend. Als Kind hat Parturiens Scharlach und Diphtherie durchgemacht, will aber sonst - abgesehen von Gelbsucht im 18. Jahre - stets gesund gewesen sein. - Erste Menstruation mit 17 Jahren, kehrte stets regelmässig alle 4 Wochen wieder, dauerte meist 3 Tage, verlief schwach und ohne Schmerzen.

Erste Schwangerschaft und Geburt verliefen normal, ebenso die zweite Schwangerschaft, Letzte Menstruation 10. Dec. 94, Conception Ende December 94, erste Kindsbewegungen Anfangs Mai 95̃ verspürt.

Webenanfang 21. IX., 2 Uhr p. m.

Status praesens: Part. $1,58 \mathrm{~m}$, ziemlich kräftig entwickelt, Temperatur und Puls normal. Brüste gut aufsitzend, Drüsenkörper gut ansgebildet, auf Druck Colostrum entleerend. Leib von kugeliger Gestalt, Nabel verstrichen, Mittellinie pigmentirt, alte und frische Striae rorhanden. Bauchdecken mässig straff.

Acusserer Befund: Kopf des Kindes scheinbar fest im Beckeneingang, der Rücken liegt links und verläuft schräg von links oben nach rechts unten, kleine Theile rechts seitlich und oben im Fundus. Herztöne rechts handbreit vom Nabel entfernt deutlich hörbar, Frequenz $12: 11: 12$. Wehen kräftig und sehr schmerzhaft.

\begin{tabular}{|c|c|c|}
\hline Beckenmaasse: & Dist. spin. & 27,0 \\
\hline & $\begin{array}{l}\text { crist. } \\
\text { Conj. extern. } \\
\text { diag. }\end{array}$ & $\begin{array}{c}30,5 \\
18,5 \\
?\end{array}$ \\
\hline Beckenumfang & & 91. \\
\hline
\end{tabular}

Innerer Befund: 2 Uhr 30 Min. p. m. Scheide weit, glatt, Muttermund fast völlig erweitert, Blase steht. Vorliegender. Theil: Gesicht; Kinn rechts, Stirn links, Gesichtslinie im queren Durchmesser des Beckeneingangs, Kopf unch etwas verschiebbar. 
Diagnose: I. Gesichtslage, Kinn seitlich, normales Ende der Schwangerschaft, Schluss der Eröffnungsperiode, Kind lebt, Blase steht. Plattes Becken.

Geburtsverlauf: 3 Uhr p. m. Die Blase wird künstlich gesprengt, es entleert sich klares Fruchtwasser.

3 Uhr 30 Minuten, Stat. idem. Es wird nun in Chloroformnarkose (Tropfmethode) durch Ass-Arzt Dr. Brïnings versucht, die Gesichtslage in eine Hinterhauptslage mittels der von Thorn modificirten Schatz-Baudelocque'schen combinirten Methode umzuwandeln; der schrägliegende Rücken wird durch Druck gegen die Schulter und Zug. am Steiss gestreckt, worauf die innen eingeführte Hand den Hinterkopf fasst, das Kinn nach oben presst und das Hinterhaupt in den Beckeneingang gleiten lässt. Hierauf wird der Kopf für einige Zeit im Becken fixirt und die Kreissende auf die linke Seite gelegt. Wehen wurden nun sehr kräftig. Schon 3 Uhr 40 Minuten wird der Kopf in der Vulva sichtbar. Nach eimigen kräftigen Wehen schneidet das Gesicht über den Damm, das Hinterhaupt stemmt sich gegen die Symphyse an und der Kopf wird geboren. Die Nabelschnur wird, da sie einmal um den Hals des Kindes geschlungen ist, gelockert und über den Kopf zurückgestreift. Das Gesicht rotirt nun nach dem rechten Schenkel der Mutter, die linke Schulter tritt über den Damm, die rechte unter der Symphyse hervor und spontan folgt das ganze Kind, ein lebendes, ausgetragenes Mäłchen, das bald kräftig schreit. Nach Aufhören der Pulsation der Nabelschuur wird es abgenabelt. Kein Dammriss. Keine Kopfgeschwulst. Fast kein Gesichtsödem, der Kopf zeigt aber noch die typische Configuration des Gesichtslagenschädels.

Instillation zweier Tropfen einer 2 proc. Argentum nitricum-Lösung: in die Augen des Kindes.

4 Uhr 20 Minuten p. m. Spontane Ausstossung der Placenta mit der kindlichen Fläche voran. Geringe Nachblutung $(150 \mathrm{~g})$. Die Maasse des Kindes:

\begin{tabular}{|c|c|c|}
\hline $\begin{array}{l}\text { Körperlänge } \\
\text { Körpergewicht }\end{array}$ & & $\begin{array}{r}51 \mathrm{~cm} \\
3300 \mathrm{~g}\end{array}$ \\
\hline Kopfdurch messer: & Grosser querer & 10,5 \\
\hline & Kleiner & 8 \\
\hline & Grader . . & 12,5 \\
\hline & Grosser schräger & 13 \\
\hline & Kleiner $\quad "$ & 9 \\
\hline Umfang & $\cdot \cdot \cdot \cdot \cdot$ & 36 \\
\hline & $\begin{array}{l}\text { st. suboce. front. } \\
\text { riph. }\end{array}$ & $\begin{array}{l}11 \\
34.5\end{array}$ \\
\hline
\end{tabular}

Wochenbett verlief ohne jede Störung. Hutter und Kind wurden am 28. IX. gesund entlassen.

II. Journ.-No. 714. Theres Weigand, Köchin, 20 J., Ip. Eintr. 27. Sept. 95.

Anamnese: Vater starb an einer Lungenkrankheit, Mutter an einer Brustoperation, 3 Brüder leben und sind gesund. Als Kind hat sie Fleckeln und Scharlach überstanden, will aber sonst stets gesund gewesen sein. Mit 15 Jahren trat die erste Periode ein, kehrte alle 4 Wochen regelmässig wieder, von 3-4 tägiger Daucr, mitunter von Schmerzen begleitet. 
Befinden in der Schwangerschaft gut; letzte Periode am 28. December 94; Conception am 10. Januar 95. Die ersten Kindsbewegungen wurden von ihr in der 20. Woche verspürt. Wehenanfang 26. IX., 5 Uhr p. m.

Siatus praesens: Aeusserer Befund 1 Uhr 20 Minuten p. m. Part. mittelgross, mässig kräftig gebant, ohne Zeichen von Rhachitis; Brïste gut entwickelt, Warzen prominent. Abdomen kugelig, Nabel verstrichen, Mittellinie pigmentirt, Bauchdecken straff, mit zahlreichen Striae versehen.

Rücken links, kleine Theile rechts, Steiss links oben im Fundus, Kopf fest im Becken. Lage der kindlichen Körperachse: schräg von links oben nach rechts unten. Herztöne rechts von der Mittellinie, von normaler Frequenz (12:12:12).

Wehen ziemlich kräftig und regelmässig. Temperatur und Puls normal.

$$
\begin{aligned}
& \text { Beckenmaasse: D. spin. . . . } 26,0
\end{aligned}
$$

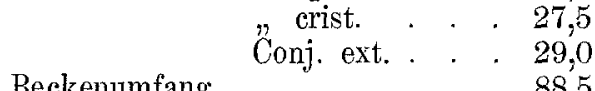

Innerer Befund 1 Uhr 20 Minuten p. m.

Scheide eng, straff, glattwandig, Frenulum erhalten, Nuttermund kleinhandtellergross, glattrandig, Blase steht noch; vorliegender Theil: Gesicht, die Gesichtslinie verläuft im queren Durchmesser des Beckeneingangs. Kinn rechts, Stirn links, Kopf noch verschieblich.

Diagnose: Ip., normales Ende der Schwangerschaft, I. Gesichtslage, Kinn seitlich, Blase steht, Muttermund fast völlig erweitert. Kind lebt.

Geburtsverlauf: Zunächst rein exspectatives Verfahren. 2 Uhr 30 Minuten erfolgt der Blasensprung. Fruchtwasser klar. Innerlicher Befund: Kinn hat sich etwas nach hinten gedreht, Gesichtslinie im rechten schrägen Durchmesser, Kopf noch verschiebbar im Beckeneingang. Es wird nun von mir der Versuch gemacht, mit 2 in die Scheide bezw. Uterus eingeführten Fingern der linken Hand, die ich an den Oberkiefer ansetzte, das Kinn über die Linea innominata zurückzuschieben, während gleichzeitig von aussen durch Herrn Dr. Brünings die rechte Schulter umfasst und nach links oben, der Steiss in umgekehrter Richtung nach rechts unten gedrängt wurde. Der Versuch musste als misslungen bezeichnet werden, da ich es wohl erreichte, das Kinn in die Höhe zu schieben, die Stirn, ja sogar die grosse Fontanelle, nicht aber das Hinterhaupt in die Führungslinie des Beckens zu leiten.

Daher wird Chloroform-Narkose eingeleitet (Verbrauch $5 \mathrm{ccm}$, schnell eintretende Toleranz, schnelles Erwachen am Schluss der Operation), in der es dureh Druck von innen auf die Stirn und Druck von aussen auf das Hinterhaupt unter gleichzeitiger äusserer Einwirkung auf Schultergürtel und Steiss unerwartet schnell, fast auf einen Rnck, gelang, das Hinterhaupt in den Beckeneingang einzustellen; in linker Seitenlage der Frau wurde es nun durch Druck von aussen fixirt, bis Part. erwachte. Sofort setzten die Wehen äusserst kräftig und häufig ein and liessen schon 3 Uhr 30 Minuten den Kopf in der Vulva erscheinen; derselbe steht im geraden Durchmesser des Beckenausgangs. 15 Minuten darauf wird er, während der Damm kräftig gestützt wird, geboren, worauf bald das ganze Kind - ein kraftiges, ausgetragenes 
Mädchen, das bald schreit - spontan ausgestossen wird. Keine Blutung. Damm intact.

4 Uhr 15 Minuten wird die Placenta spontan, mit der fötalen Fläche voran, geboren. Mässige Blutung $(250 \mathrm{~g})$. Uterus gut contrahirt. Befinden gut. Temperatur 37,4 ; Puls 84 . Instillation ron Arg. nitr. in die Augen des Kindes.

Maasse des Kindes:

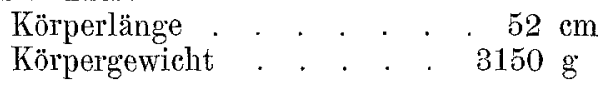

Kopfmaasse:

Grosser querer Durehm. . . . 9

Kleiner " $\quad " \quad$. . . 8

Gerader Durchm. . . . . . 12

Grosser schräger Durchm. . . 13

Kleiner " $\quad . \quad 9$

Umfang. . . . . . . . . 36

Dist. suboce. front. . . . . . 11

Periph. ". . . . . . 33

Das Wochenbett verlief in den ersten fünf Tagen ohne jede Temperatursteigerung. Am 6. Tage aber wurde des Abends 39,1 gemessen, bei einem Pulse von 96 in der Minute; 2 Stunden später 40,6;. Puls 108. Wöchnerin klagte über Kopfschmerzen; die linke Brust ist geröthet, druckempfindlich und zeigt oberflächliche Schrunden an der Warze. Nachmittags leichtes Frösteln. Linkes Parametrium etwas druckempfindlich. Leib nicht aufgetrieben. Uterns 3 Finger breit über der Symphyse. Lochialsecret serosanguinolent, nicht übelriechend, Aussengenitalien nicht geröthet. Ordinat.: Eisblase auf das Abdomen; Scheidenausspülung mit heisser 1 proc. Lysollösung. 2 mal ì $1,0 \mathrm{~g}$ Chinin. Am nächsten Morgen: Temperatur 38,0; Puls 96, Abends: 37,2, Puls 96. gutes subjectives and objectives Befinden, Temperatur blieb normal bis zum Tage der Entlassung (10 Tage post part.).

III. Der dritte Fall wurde von mir in der geburtshülflichen Poliklinik beobachtet. Am 24. September 95, 11 Uhr 30 Minuten a. m. wird von der Hebamme Frau Str... poliklinische Hülfe wegen Gesichtslage erbeten.

Bei meiner Ankunft fand ich folgenden Status:

Katharina RakI, Kellnerin, 28 J., III p., mittelgross, kräftig gebaut, will nie krank gewesen sein, hat vor 11 Jahren 1 normale Entbindung durchgemacht mit regelmässigem Wochenbett: vor 1 Jahre 1 Abort im 3. Monat, Menstruation stets regelmässig, zum letzten Mal am 19. Jan. 95. Befinden in der jetzigen Schwangerschaft gut.

Part. kreisst seit 9 Stunden. Bei der äusseren Untersuchung fand ich im Fundus uteri kleine Theile, Herztöne rechts handbreit von der Mittellinie entfernt, Frequenz $11: 12: 12$, sonst ist nicht viel deutlich abzutasten, da die Wehen sehr schnell und kräftig auf einander folgen. Ueber der Symphyse fühlt man links eine harte lkugelige Resistenz, die offenbar einem Segment des Schädels entspricht, darüber eine diesem entsprechende Furche.

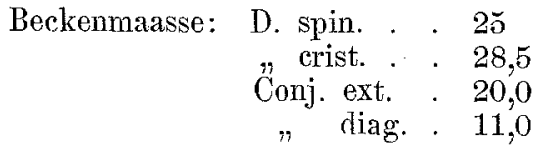


Innerer Befund: Mm. 2-3-markstückgross, Blase gesprungen (nach Angabe der Kreissenden vor ó Stunden); Stirn links vorn, Kinn rechts hinten, Gesichtslinie im rechten schrägen Durehmesser, Kopf im Beckeneingang scheinbar fest.

Da ich nur die Instruction hatte, bei Gesichtslage möglichst exspectativ zu verfahren, so fragte ich telephonisch Assistenzarzt Dr. I üller an, ob ich die Umwandlung in Hinterhauptslage machen solle. Er gab seine Einwilligung und sandte mir mit dem Praktikanten der Poliklinik einen Colpeurynter, den ich, falls die Operation misslingen sollte, verwenden könnte. 12 Uhr 30 Minuten p. m. Nach Katheterismus der Blase und Eintritt tiefer Chloroform-Narcose ging ich zuerst mit 2 Fingern der linken Hand (später mit der halben Hand) in die Scheide ein, drückte den Oberkiefer gegen die Brust des Kindes hinauf, umfasste dann das Hinterhaupt, drückte es herab (worauf sofort das Kinn von der rechten Linea innominata wegrückte und die grosse Fontanelle den Fingern entgegenkam), während gleichzeitig von aussen, nach Schatz, die rechte kindliche Schulter nach links und etwas nach oben, der Steiss nach rechts und etwas nach unten gedrängt wurde. Die Umwandlung volizog sich sofort, unter einer deutlich wahrnehmbaren, einschnappenden Bewegung des Kopfes, so dass ich bald die grosse Fontanelle rechts, die kleine links füblte (Pfeilnaht fast quer verlaufend). Dìe Herztöne, die vorher rechts zu hören waren, konnten jetzt deutlich links gehört werden. Dr. Müller, der jetzt eintrat, liess die Frau auf die linke Seite legen und wir fixirten das Hinterhaupt von aussen, in der gewomnenen Stellung, bis Part. erwachte (nach ca. 4 Minuten) und kräftige Wehen einsetzten. 1 Uhr 30 Minuten, also eine Stunde später, war der Muttermund bereits völlig erweitert, da die Wehen sehr kräftig und stürmisch wurden. Kopf im rechten schrägen Durchmesser in Beckenweite. Herztöne gut. In Beckenenge hält er sich so lange auf, dass, da der Kristeller'sche Handgriff nicht zum Ziele führte, ich die Vorbereitungen zur Zange treffen musste, zumal die Herztöne jetzt sehr dumpf und unregelmässig wurden. Doch kam der Kopf 2 Uhr 50 Minuten unter stürmischen Wehen endlich zum Einschneiden und wurde 10 Minuten später völlig geboren, mit der nächsten Wehe der ganze, bald kräftig schreiende, reife Knabe von $54,5 \mathrm{~cm}$ Länge und $36 \mathrm{~cm}$ Kopfumfang. 20 Minuten darauf spontane Geburt der Placenta. Damm intact, Uterus gut contrahirt, Blatung unerheblich. Heisse 1 proc. Lysolausspülung. Der Kopf des Kindes zeigt typische Deflexionshaltung und Andeutung von Struma, ist aber nicht in der für Gesichtslage sonst charakteristischen Weise ausgezogen. Das Wochenbett verlief ohne jede Störung. Mutter und Kind, das sehr gut gedeiht, werden am 8. Tage ans der Behandlung entlassen.

Im ersten dieser Fälle hatte Part. seit 2 Uhr a. m., also schon $15^{1 / 2}$ Stunden gekreisst; Muttermund war fast völlig erweitert, und da trotz vorausgeschickter künstlicher Blasensprengung, mit der man ein Tiefertreten des vorliegenden Theiles beabsichtigte, das Gesicht im Beckeneingang verharrte, so wurde bei seitlich stehendem Kinn zur manuellen Umwandlung nach Schatz-Thorn geschritten:

Ein derartiger Eingriff mag schon auf den ersten Blick in diesem Falle nicht indicirt erscheinen; und es muss zugegeben 
werden, dass or thatsächlich nicht indicirt gewesen ist. Der Vorwurf der Polypragmasie, den man gegen ein so actives Vorgehen in der Behandlung einer uncomplicirten Gesichtslage erheben kann, müsste als berechtigt angesehen werden, wenn man in allen derartigen Fällen ebenso voreilig den Naturkräften vorarbeiten wollte. In diesem Falle aber handelte es sich lediglich um einen Versuch, um die Ausnützung der Gelegenheit, die von Thorn erst jüngst von neuem empfohlene Methode auf ihren Werth zu prüfen. Die Berechtigung zu derartigen Versuchen wird einer Klinik Jeder zugestehen müssen, um so mehr, je ungefährlicher sie sind.

Dass aber in der That die Umwandlung der Kreissenden keinen Schaden gebracht hat, lehrt der weitere Verlauf: dem schon 15 Minuten nach erfolgter Umwandlung wurde das sehr kräftige Kind spontan geboren.

Die zweite Parturiens hatte ein etwas verengtes Becken; sie Kreisste seit $21 \frac{1}{2}$ Stunden. Muttermund war völlig erweitert, Blase gesprungen; da das Kinn sich im weiteren Verlauf naclı hinten gedreht hatte, und die Geburt keine Fortschritte machte, so wird dieselbe Methode der Umwandlung mit bestem Resultat ausgeführt; denn die sofort kräftig einsetzenden Wehen führten nach $11 / 4$ Stunden zur spontanen Geburt des Kindes.

Im dritten, poliklinisch behandelten Falle, hatte die Eröffnungsperiode schon vor 12 Stunden begonnen. Muttermund war etwa 3 Markstück gross, die Blase vor $71 / 2$ Stunden gesprungen. Das Kinn war auch hier nach hinten gerichtet. Auch hier gelang: die manuelle Umwandlung in Hinterhauptslage überrasehend leicht; der Erfolg war der, dass nach $21 / 2$ Stunden das Kind lebend spon$\tan$ geboren wurde.

Wenn sich hier der Austritt des Kopfes verzögerte, so lag dies an dem Missverhältniss zwischen jhm und dem Becken. Der Schädel des kräftigen Kindes ( $54,5 \mathrm{~cm}$ lang) hatte nämlich einen Umfang von 36, während die Beckenenge in der Gegend der Spinae und des Beçrenausgangs eine Verengerung zeigten. (Leider ist Wöchnerin meinem Wunsche, sich in der Klinik vorzustellen, behufs Feststellung der Beckenausgangsmaasse, nicht nachgekommen.)

Das Auftreten einer hohen Temperatursteigerung im Wochenbett (Fall 2) ist natürlich nicht der Methode zur Last zu legen, sondern einem Fehler: in der Antiseptik, der jedoch zu einer Zeit, wo auch Hebammenschülerinnen untersuchen (ich gestattete zwei 
Schülerinnen die innere Untersuchung, um ihnen die seltene Lage zu demonstriren, konnte aber ihre Desinfection nicht streng überwachen, da ich in demselben Saale noch mehrere Geburten zu leiten hatte) wohl rorkommen und schon bei der gewöhnlichen Exploration die Kreissende inficiren kann. Ueberdies hatte ja die Infection keine üblen Folgen; denn sehon am nächsten Tage fiel dic Temperatur wieder zur Norm zurück, eine Erscheinung, dio ja nicht selten bei Wöchnerinnen, auch solchen, die ganz normal geboren haben, beobachtet wird und vielleicht auf einem saprämischen Process beruht, dessen Möglichkeit nach den neuesten Untersuchungen von Kroenig, Menge und Doederlein über das Scheidensekret Schwangerer nicht von der Hand gewiesen werden kann.

Betrachten wir nun zunächst die Indication, welche in den beiden letzten Fällen den Eingriff rechtfertigte, so war dieser wegen pathologischer Einstellung des Kinns nach hinten vorgenommen worden.

Thorn hält nun den Versuch der manuellen Umwandlung der Gesichtslagen „eventuell unter Zuhülfenahme der Narkose" dann für angezeigt, wenn "gegen Ende der ersten oder im Laufe der zweiten Geburtsperiode eine Verzögerung der Geburt eintritt, die bei längerer Dauer ernste Befürehtungen für das kindliche Leben aufkommen lässt" und fährt dann fort: "Gleichgültig ist dabei die Stellung des Kinns; Vorbedingungen sind eine genügende Beweglichkeit der Frucht and eine zur Einführung der halben Hand genügende Erwreiterung des Muttermunds."

Thorn verwirft also den prophylakischen Standpunkt vollkommen und lässt sich nur von der Rücksichtnahme auf das Leben des Kindes leiten, ohne auch für die Mutter die Vortheile einer, wic er selbst sagt, ungefährlichen, schonenden und leicht ausführbaren Operation auszunützen. Wir selbst können nur bestätigen, dass der Eingriff in Narkose überraschend leicht gelingt, dass der Schädel wie mit einem Ruck sich in das Becken einstellt, ähnlich wie der: luxirte Humeruskopf mit einer schnappenden Bewegung in die Gelenkpfanne einspringt. Sofort nach erfolgter Umwandlung konnten wir ebenso wie Thorn, Ziegenspeck, Brennecke, Wullstein, v. Weiss constatiren, dass die Wehenthätigkeit fast momentan eine bessere wurde; wurde doch in unserem ersten Falle die Geburt schon nach einer Viertelstunde spontan beendigt!

Der günstige Einfluss auf das schnelle Fortschreiten der Ge- 
burt erklärt sich daraus, dass die Wehen, die bei Gesichtslage oft stürmisch und krampfartig und häufig sehr schmerzhaft sind, wcil das Hinterhaupt die Uteruswand stark dehnt, regelmässiger aufeinanderfolgen und auf das Hinterhaupt auch intensiver wirken können, während bei der Einwirkung auf den Gesichtslagenschädel ein Theil ihrer Kraft verloren geht.

Warum num Thorn angesichts der guten Erfolge seiner Methode für dieselbe so enge Indicationen aufgestellt hat, kann ich nicht cinsehen. Ich möchte vielmehr auf Grund der bisher veröffentlichten Fälle und unserer drei Beobachtungen für eine prophylaktische Umwandlung der Gesichtslagen warm eintreten, sie also schon dann vornehmen, wenn die Ausführung möglich ist und zwar unter folgenden Verhältnissen:

Handelt es sich um eine sog. primäre Gesichtslage, so würde ich, wie es Schatz vorgeschlagen hat, versuchen, die Umwandlung möglichst noch in der Schwangerschaft nach seiner Methode, also lediglich durch äussere Handgriffe in Schädellage umzuwandeln. Ich möchte vorschlagen, bei Ausführung der Umwandlung an der Schwangeren, also bei nicht genügend durchgängiger Cervix vom vorderen oder hinteren Scheidengewölbe aus auf den Kopf einzuwirken, und dadureh die äusseren Handgriffe zu unterstützen. Ich habe zwar über diese Modification keine Erfahrung, halte sie aber für ausführbar und unter antiseptischen Cautelen ausgeführt für ungefährlich.

Misslingt die äussere Umwandlung nach Schatz, so hat man wenigstens in nichts geschadet.

Hat aber die Eröffnungsperiode schon begonnen, so wird man etwa nach folgenden Gesichtspunkten zu verfahren haben.

Zunächst ist die Frage zu entscheiden, ob der Kopf noch beweglich ist. Dies ist meiner Ansicht nach oft nur in Narkose mit Sicherheit zu entscheiden. Denn mitunter wird der mit dem Gesicht scheinbar tief im Becken stehende, mit seiner grössten Circumferenz thatsächlich noch im Beckeneingang befindliche Schädel eine Unbeweglichkeit vortäuschen, während in Narkose eine Zurückschiebung desselben über die Linea innominata noch sehr gut ausführbar ist. Diese Erfahrung konnte ich im Fall III. machen.

Ist der Schädel noch beweglich, der Muttermund etwa für 2 bis 3 Finger durehgängig, so steht, falls ein normales oder; was gerade bei Gesichtslagen häufig der Fall ist, ein plattes Becken vorliegt, dem Versuch der Umwandlung nichts entgegen. 
Ob die Blase schon gesprungen ist, oder noch steht, ist wohl gleichgültig; der Arzt wird meist erst nach dem Blasensprung gegerufen werden; zumal dieser, wie die Erfahrung gezeigt hat, gerade bei Gesichtslage vorzeitig zu erfolgen pflegt.

Was nun die Stellung des Kimns betrifft, so kann ich Thorn nicht Recht geben, wenn er dieselbe für gleichgültig hält, falls nach ihm die Indication zur Umwandlung der Gesichtslage gegeben ist. Wohl kann man bei mento-posteriorer Gesichtslage durch seine Methode eine Hinterhauptslage erzielen. Auch bei seitlichem Stande des Kinns resultirt nach gelungener Reposition eine physiologische Lage, nämlich Querstand des Kopfes im Beckeneingang; also wird man dann z. B. bei I.Schädellage die kleine Fontanelle links, die grosse rechts, die Pfeilnaht fast quer verlaufend vorfinden; und schon bei entsprechender Lagerung auf die Seite der kleinen Fontanelle, also auf die linke, wird das Hinterhaupt tiefer und nach vorn treten.

Anders aber liegen die Verhältnisse bei mento-anteriorer Gesichtslage: wenn auch hier Thorn die Umwandlung bei einer für das Kind gefahrdrohenden Geburtsverzögerung vornehmen will, so ist das, wie ich glaube, ein Fehler; denn da das Kinn nach vorm gerichtet ist (der Rücken des Kindes also nach hinten), so kann durch die manuelle Umwandlung, wie man sich leicht auch am Phantom überzeugen kann, nie eine Hinterhauptslage, sondern nur eine Vorderhauptslage entstehen. Von dieser aber wissen wir, dass sie weder schneller, noch erheblich günstiger für das Kind verläuft. Tritt bei mento-anteriorer Gesichtslage, die man ebenso wie die Vorderhauptslage unter uncomplicirten Verhältnissen den Naturkräften überlassen kann, eine Indication zur raschen Beendigung der Geburt ein, so wird man den Muttermund, wenn er nicht genügend erweitert ist, durch Einlegen eines Colpeurynters, event. bei hochgradiger Gefahr durch Dührssen'sche Incisionen für die Wendung auf beide Füsse und Extraction (bei hochstehendem bebeweglichen Kopf), für die Application der Zange bei fest eingetretenem Kopf vorbereiten.

Bezüglich der Ausführung der Operation selbst möchte ich noch einige Punkte hervorheben, die mir wichtig, aber in den bisherigen Veröffentlichungen nicht genügend betont erscheinen.

Die Narkose halte ich für einen raschen Erfolg der Operation für unerlässlich, schon deshalb, weil die Entscheidung der Frage, ob die Frucht noch beweglich ist oder nicht, oft nur in Narcose 
möglich ist. Auch hat ja die Erfahrung gelehrt, dass gerade Kreissende die Narkose auffallend gut vertragen, bei Anwendung schon ganz geringer Mengen Chloroforms schnell in das Stadium der Toleranz kommen und auch schnell aus der Narkose erwachen. Auf die Frucht hat die Narkose ebenfalls keinen schädigenden Einfluss; denn wenn auch Budin nach mehrstündiger Chloroformnarkose an 2 Kreissenden bei deren Neugeborenen eine gewisse Schlafsucht constatiren konnte, und Zwejfel den Nachweis von Chloroform im Blute von Neugeborenen, deren Mütter in Chloroformnarkose entbunden waren, erbracht hat, so sind die aufgenommenen Mengen - wenn bei einer kurzdauernden Narkose überhaupt etwas auf die Frucht übergeht - doch so gering, dass man keinen Schaden darans zu befürchten braucht.

Indessen kann man es begreifen, wenn ängstliche Gemüther erst einen Versuch machen wollen, die Umwandlung an der nicht narkotisirten Frau zu machen. Alsdann darf man aber, wenn die Methode nicht gleich zum Ziele führt, keine Gewalt anwenden. Ferner halte ich es für ausserordentlich wichtig, bei Ausführung der combinirten manuellen Umwandlung stets eine gewisse Reihenfolge derart inne zu halten, dass man mit den schonenderen Handgriffen, die oft spielend leicht zum Ziele führen, beginnt und erst dann zu den eingreifenderen übergeht. So möchte ich folgenden Gang in der Ausführung dieser Operation als rationell empfehlen. Nachdem man die narkotisirte Kreissende katheterisirt hat, lagert man (Querbett ist nicht erforderlich) sie nach dem Vorschlage von Thorn auf die dem Kinn entsprechende, also bei I.Gesichtslage auf die rechte Seite und geht nun in die Scheide mit der halben, nöthigenfalls mit der ganzen Hand ein, mit zwei Fingern in den Uterus, setzt sie an den Oberkiefer an und führt, während gleichzeitig ein Assistent oder die Hebamme die kindliche Schulter nach der Rückenseite des Kindes und nach oben, den Steiss in umgekehrter Richtung nach abwärts drückt, folgende Bewegungen aus: man sucht, während man von aussen das Hinterhaupt kräftig in das Becken drückt, den Oberkiefer, dann die Stirn, dann die grosse Fontanelle in die Höhe zu schieben und schliesslich umfasst man mit beiden Fingern das Hinterhaupt, wenn es unter den bisberigen Manipulationen noch nicht in den Beckeneingang eingetreten wäre. Sollte auch jetzt die Umwandlung noch keine vollständige sein, so desinficire man sich noch einmal, erfasse das Hinterhaupt 
mit der diesem entsprechenden Hand und ziehe es in das Becken hinein.

Ich lege deshalb Werth darauf, zuerst an den Oberkiefer zu gehen, weil ja das Gesicht zum Theil schon unterhalb des Beckeneingangs (wie in Fall II) stehen kann; wollte man in dieser Stellung das Hinterhaupt umfassen and den Schädel um seine Queraxe zu rotiren versuchen, so würde die Umwandlung mindestens sehr erschwert, wenn nicht unmöglich werden.

Warnen möchte ich davor, die Finger an den Unterkiefer anzusetzen; denn durch diese Manipulation würde man die Frucht zu vorzeitigen Schluckbewegungen veranlassen. Auch hat man bei dem Zurückschieben des, Gesichts jeden Druck auf die Augen des Kindes zu vermeiden.

Nach gelungener Umwandlung ist die Kreissende auf die Seite der kleinen Fontanelle zu lagern und von aussen das Hinterhaupt so lange in das Becken zu drücken, bis kräftig einsetzende Wehen dasselbe in der gewonnenen Lage fixiren.

Fassen wir nun unsere Betrachtungen noch eimmal kurz zusammen, so ergeben sjch folgende Sätze:

1. Bei primären Gesichtslagen, d. h. den schon in der Schwangerschaft bestehenden, ist die prophylaktische Umwandlung in Schädellage nach der Methode von Schatz zu versuchen.

2. Bei Gesichtslagen, bei denen in der Eröffnungsperiode das Kinn hinten steht, ist der Versuch der Umwandlung in Hinterhauptslage nach der combiuirten Methode von Schatz-Thorn zu versuchen, wenn

a) der Muttermund für 2-3 Finger durchgängig,

b) das Becken normal oder mässig verengt ist,

c) die Frucht noch genügende Beweglichkeit zeigt.

3. Die Narkose ist bei den sub 2 aufgeführten Fällen sofort einzuleiten, wenn ein schonender Versuch an der nicht narkotisirten Frau misslungen ist.

4. Contraindicirt ist der Versuch der Umwandlung dann, wenn Gefahr für Mutter oder Kind besteht, die eine rasche Beendigung der Geburt erfordert.

Freilich wird in manchen Fällen die Umwandlung unmöglich sein z. B. bei tiefem Sitz der Placenta, bei Geschwülsten der Gebärmutter u. dergl.; da sich aber derartige Fälle von vornherein nicht diagnosticiren lassen, so ist der Versuch der manuellen Um- 
wandlung in Hinterhauptslage zu machen; seheitert er, so hat sich dic weitere Behandlung nach den heute allgemein anerkannten Grundsätzen einer exspectativen Therapie zu. richten.

Jch schliesse meine Betrachtungen mit dem Wunsch, dass diese Methode nach obigen Principien weiter geübt und jede einsehlägige Beobachtung veröffentlicht werden möge, damit man aus einer grösseren Reihe von Fällen zu einer sieheren Entscheidung der Frage gelangen kann, ob die Methode in Stande ist, die Prognose der Gesichtslagen für Mutter und Kind zu bessern, ob sie es verdient, in den Lehrbüchern und Operationskursen in die allgemein geburtshülflichen Verfahren gleichberechtigt cingereiht zu werden.

Zum Schlusse dieser Arbeit komme ich noch der angenehmen Verpflichtung nach, meinem hochverehrten Lehrer und Chef, Herrn Geh. Medicinalrath Prof. v. Winckel für die gütige Ueberlassung der Krankengeschichten meinen verbindlichsten Dank auszusprechen.

\section{Literatur.}

Breunecke, Berlin. klin. Wochenschrift. 1882. No. 4. Doederlein, Leitfaden f. d. geburtsh. Operationscurs. Leipzig 1895.

Fritsch, Berl. klin. Wochenschr. 1872. No. 32.

Gossmann, Münch. med. Wochenschrift. 1895. No. 23.

Kaltenbach, Lehrbuch der Geburtshälfe.

Kehrer, Lehrbuch der operativen Geburtshülfe. Stuttgart 1891.

Ko losser, Ueber Gesichtslagen und deren manuelle Umwandlung in H. Inaug.-

Diss. Halle 1880.

Pippingsköld, Berl. B. z. Geb. u. Gyn. I. S. 279 .

Runge, Lehrb. d. Geburtshülfe.

Schatz, Arch. f. Gynäkolog. B. V. S. 306.

Schatz, Arch. f. Gynäliolog. B. XXVIT. S. 293.

Schröder, Lehrb. A. Geburtshülfe.

v. Steinbüchel, Ueber Gesichts- und Stirnlagen. Altr. Hölder. Wien 1894.

Thorn, Zeitschrift f. Geburtshülfe and Gynäk. Bd. XIII.

Thorn, Zeitschrift f. Geburtshülfe und Gynäk. Bd. XXXI. 1895.

v. Weiss, Volkmann's klin. Vorträge. 1893. No. 74.

Welponor, Arch. f. Gynäk. Bd. XI.

v. Winckel, Klin. Beobachtungen zur Pathologie der Geburt. Rostock 1869.

r. Winckel, Lehrbuch d. Geburtshülfe. Leipzig 1893.

Ziegenspeck, Volkmann's klin. Vorträge. No. 284.

7weifel, Jehrbuch d. Geburtshülfe. 